\title{
Development of Workshop Environment for Supporting Collaborative Problem Framing
}

\author{
Shinnosuke WANAKA ${ }^{\mathrm{a}, 1}$, Kazuo HIEKATA ${ }^{\mathrm{a}}$ and Bryan R. MOSER ${ }^{\mathrm{ab}}$ \\ a The University of Tokyo, Japan \\ ${ }^{\mathrm{b}}$ Massachusetts Institute of Technology, USA
}

\begin{abstract}
The objective of this paper is to develop a collaborative workshop environment for decision-making to improve collaboration in a cross-cultural team. Final goal of this research is to improve collaboration in a cross-cultural team, and this paper approaches a problem of communication and group performance among the team members during problem framing stage. To achieve the goal, we utilized systems approach and workshop monitoring system. Systems approach is utilized to fill the gap between the cross-cultural team members and to improve the communication efficiency. To make it applicable to non-experts of system modeling, an information system to support the modeling tasks is developed. The workshop monitoring system accumulates log data related to the collaboration, and provides feedback about the teamwork during the workshop. In a case study, as a part of demonstration of the proposed workshop environment, an experiment to show the usefulness of the monitoring and automatic visualization system is conducted.
\end{abstract}

Keywords. Computer-supported Collaborative Work, Transdisciplinary Teamwork, System Modeling

\section{Introduction}

Industry in these days can be represented as sociotechnical system [1], in which not only technical systems but also social systems highly combine with each other. Technology and society are rapidly changing, and various kinds of systems in industry should be redesigned for the changes. On the other hand, design of system of systems (SoS) is not easy for an individual designer because the system is a gathering of multidomain systems and too complicated for the individual designer to understand. Moreover, external environment of the system is also changing rapidly, and new situation people have not yet experienced happens. Therefore, it is not possible to design the system only by experts' experience and intuition, which are usually considered to be useful for a complex, but static system design. Collaborative design by multiple domain experts can be a solution. However, as for the collaborative design, several papers reported some problems as well as some advantages [2]. This paper especially focuses on 2 kinds of problems, a communication problem across different domains and a problem of group performance.

\footnotetext{
${ }^{1}$ Corresponding Author, Email: swanaka@s.h.k.u-tokyo.ac.jp.
} 
In the collaborative design with multiple domain experts, it is needed to care about the gap of background and assumption between different domains. System model is a way to represent a system without depending on the system's domain [3], and it can be utilized for problem framing of design process and useful way to share knowledge among the design project [4]. However, it is tough for non-experts of system modeling to describe system model by the modeling language, and it takes a long time to learn the methods and obtain skill set to be a system modeler. In short, the systems approach which is a design method using system model is considered as a useful way to fill the gap between different domain's background. However, it also has the difficulty to apply to a normal design group whose members are not experts of system modeling.

As another aspect, we need to take care about group performance in teamwork. This is mainly studied by cognitive science, and they reported several phenomena which shows group has different performance from the gathering of individuals. For example, collective intelligence [5] shows that groups have intelligence as well as individual, but not correlated to members intelligence directly. Groupthink [6] is a psychological phenomenon that occurs within a group of people in which the desire for harmony or conformity in the group results in an irrational or dysfunctional decisionmaking outcome. Collective intelligence is correlated to mainly 3 things, average social sensitivity of group members, variance in the number of speaking turns, and proportion of females in the group. Groupthink is mainly caused by high group cohesiveness, group with detective leaders, and situational context like high stake decisions and time pressure and so on. These are just 2 examples picked up in the various kinds of researches, but they are related to a similar factor, conversation in the group. In summary, teamwork has several potential risks to decrease the performance for decision-making, and distribution of conversation and sharing opinion is one of the impactful elements for the group performance.

The objective of this paper is to develop a collaborative workshop environment for decision-making to improve collaboration in a cross-cultural team. This paper especially approaches 2 kinds of problems, communication among the team members and group performance. To solve the problems, a concept of instrumented workshop environment is proposed, in which systems approach is utilized to fill the gap of their background, and monitoring and automatic visualization system of conversation amount is applied to improve the group performance. In case study, as a part of demonstration of the proposed environment, an experiment to show the usefulness of the monitoring and automatic visualization system is conducted.

\section{Proposed collaborative design workshop environment}

\subsection{Overview}

This section proposes a framework of collaborative design workshop environment which is based on systems approach but designed to be executable by non-experts of system modeling, and care about the group's performance. Figure 1 shows an overview of the proposed workshop environment. First the environment assumes a specific system design protocol based on the existing systems approach. The design protocol defines procedures how teams frame their problems by using system model. And the workshop environment provides them sharable workspace, support functions to execute the protocol, and monitoring and feedback system of conversation in the workshop. As 
mentioned in the introduction, conversation is one of the key factors to indicate group performance. This is the reason why the system monitors and generates a feedback information. Supporting by these 3 functions enables teams to execute collaborative workshop of problem framing. In the workshop, they explicitly express their thought, idea, and mental model as system models, and monitoring and feedback system of conversation amount always attempts to bring out every member's opinions and prevent biased conversation which is dominated by specific people.

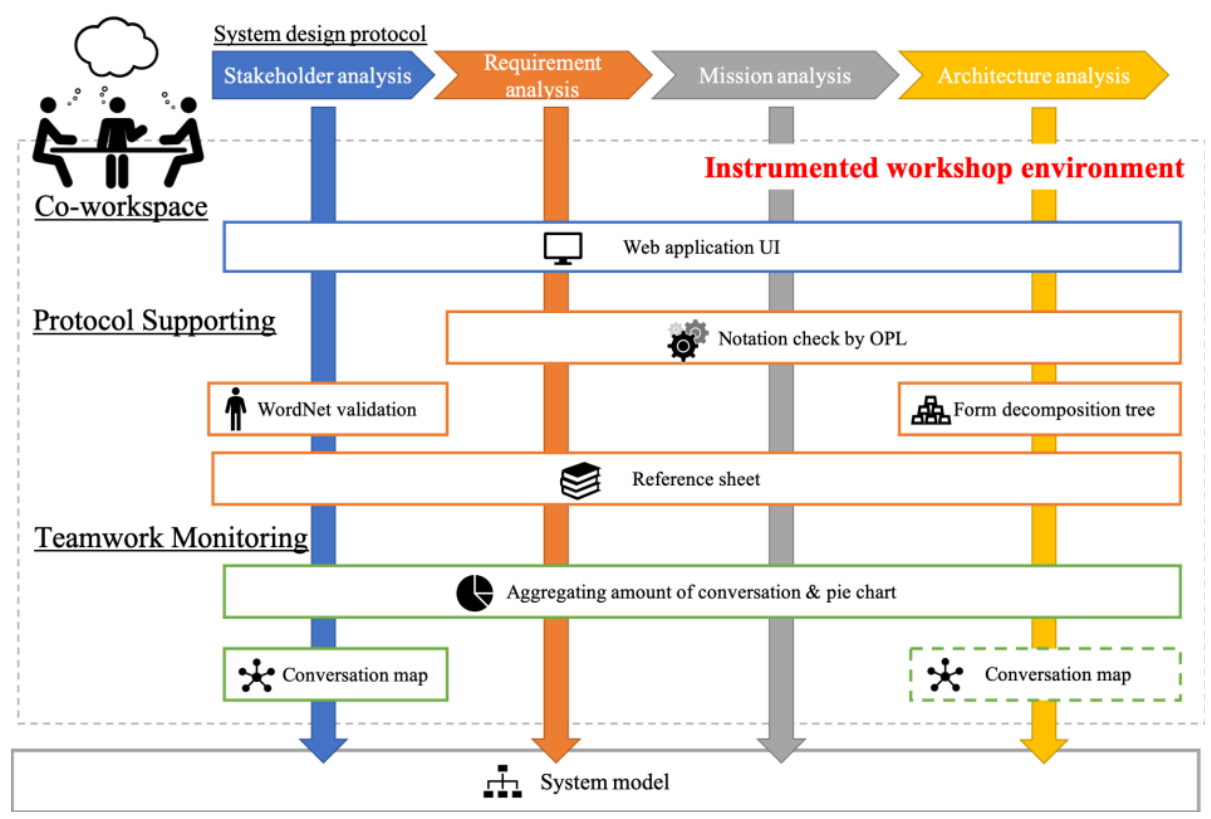

Figure 1. Overview of the proposed workshop environment.

\subsection{System design protocol}

The design process itself consists of 7 steps, stakeholder analysis, requirement analysis, system mission analysis, system architecture analysis, enumeration of alternative options, building a calculation model and tradespace analysis.

In stakeholder analysis, stakeholders of design projects and their relationship are analyzed by stakeholder value network (SVN) [8], and it is clarified that value the project will provide, who is a beneficiary of the value and what is need of the beneficiary. In requirement analysis, the need defined in the stakeholder analysis is interpreted and decomposed into 2 parts, functional and non-functional requirements. Functional requirement is minimum requirement system should achieve, and nonfunctional requirement is system's lifecycle properties and performance related requirement. And then, analysis and description about how the requirement is fulfilled by the current system. This is system mission analysis, in which the current system's mission clarified as intent, function and form. Also, non-functional requirements are translated into more concrete performance indexes. In system architecture analysis, designers try to dig in more detail about the system's function. During the step, system's function is decomposed into several internal functions and mechanism about how the 
function emerges in the system. As a result of system architecture analysis, a long list of internal functions and the structure are obtained. On the basis of the result, exploration of alternative options is conducted. This is a kind of ideation process. All the alternative options to change system's configuration can be represented by switching, adding or removing system functions or forms on the result of system architecture analysis. Next step is building calculation model to evaluate the enumerated options in the previous step. Any type of calculation is fine to evaluate them, for example, simplified mathematical equation, agent-based simulation and so on. Requirement for the calculation model is that it should evaluate all the enumerated options and the performance indexes should be based on indexes clarified in the system mission analysis. Finally, designer can obtain the result of the evaluation. The evaluation result by multiple performance indexes constructs tradespace which shows trade-off relationship among the indexes and how they are related with each other. From the tradespace, Pareto optimal designs can be extracted, and designers can make a decision which should be their design on the basis of the Pareto optimal solutions and their preference for the performance.

4 steps from stakeholder analysis to system architecture analysis are analysis of the current situation, and knowledge clarification and sharing process. This paper proposed the entire design process but focus on these 4 steps. We assumed the design process and developed the collaborative workshop protocol. Rest of this section explain how people execute those processes as collaborative works, the method, notation, and procedure.

\subsection{Instrumented workshop system}

System design protocol assumed in this paper is mentioned in 2.2. It is based on the existing systems approach but simplified and limited to specific protocol so that it is easy to be applied for collaborative workshop by people who are not experts of systems approach. However, it still contains difficulties for the people to utilize. Instrumented workshop system provides several functionalities to support to execute the system design protocol like user interface, automatic validation of system model's notation, and so on. The overview of the developed system is shown in Figure 2.

First, the system has application which provides some UIs as workspace during workshop. The UI is editing tool and helps people to describe system models. And the application connects to 2 other systems, reference sheets and notation checker, both are supporting system to the system model description. Reference sheet is summary of vocabulary utilized in the system design protocol and provides a word search function.

The vocabulary is not so familiar with the non-experts of system modeling. However, the reference sheet provides easy access to the vocabulary's definition. Notation checker is useful to prevent incorrect use of system model's notation. The target users of the system are not experts of system modeling so that they sometimes make a mistake to describe their model. For example, stakeholder in SVN should be person or organization, but some people try to put product or service as stakeholder in SVN. To prevent this kind of incorrect usage of system model, system automatically check the model people built and feedback to the users. These are the functionality to support users directly to build their system model. Moreover, this workshop is collaborative group work and as mentioned in related works, it is needed to consider the group's performance as well. Some previous researches show that the equality of conversation's turn taking is one of the most impactful factors for group's performance. 
Automatic conversation visualizer provides automatic visualization of conversational distribution during the workshop and provides feedback to the group in real time. By the automatic visualization, users are always able to take care of the balance of conversation and prevent unexpected decline of the group's performance.

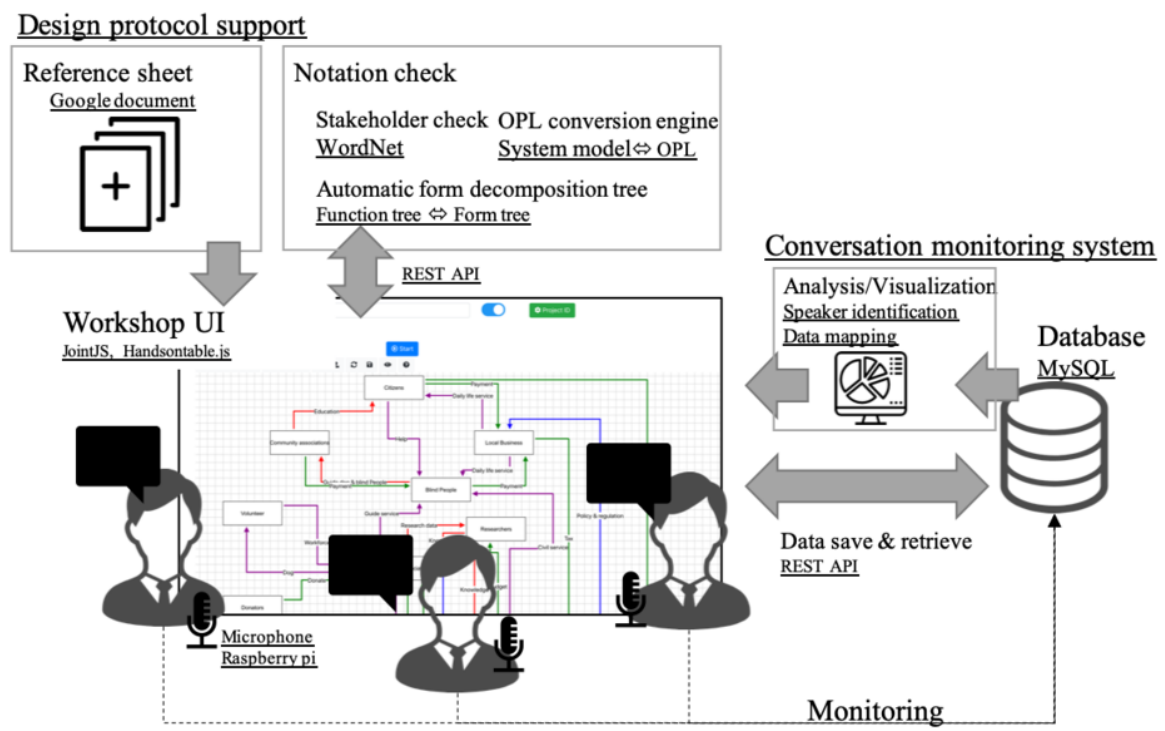

Figure 2. Overview of the developed instrumented workshop system.

\subsubsection{User interface for the system design protocol}

UI provides functions to edit system model on the web application. By using this UI, users can make such a diagram in beautiful and quick way. Moreover, UI has another important function, users' action logger. If the UI is introduced to the workshop, it will accumulate users' action like adding nodes, arcs, deleting, change text, and so on. This $\log$ data is essential for visualization of conversation amount.

\subsubsection{Support methods for the system design protocol}

This section explains 2 kinds of support methods, reference sheet and notation checker in more detail. Reference sheet is summary of vocabulary's definition and notation of system model used in this workshop. Reference sheet is developed by Google document and released on the web. Users can search words they want to know and get the definition and notation. As for the notation checker, different functionalities are implemented for each step of design protocol.

\subsubsection{Notation check for stakeholder analysis}

In SVN of stakeholder analysis, the stakeholders should be person or organization. However, through a bunch of preliminary workshops, it is found that people sometimes include product or service as the stakeholders. To check this mistake, the developed system utilizes Wordnet [9], a large lexical database of English, and alerts when stakeholders users put into the UI are not person or organization. In more detail, 
WordNet has information about class of words, for example, artifact, cognition, person, group and so on. The system sends the word inputted as stakeholder to service server, and retrieve the class information. If the word has artifact class, the system detects it might be product or service and alerts it to users.

\subsubsection{Notation check for requirement analysis, system mission analysis, and system architecture analysis}

In the requirement analysis and system mission analysis, users describe the system's requirement by functional requirement and performance requirement. However, they are not familiar with non-experts of system modeling and it may cause incorrect usage of the words. The template defined in this paper for requirement analysis can be transformed to object process methodology (OPM) format, and OPM has own validation framework, object process language (OPL). OPM is one of the most famous modeling languages and OPL is a natural language expression of OPM model[10]. Natural language is one of the most familiar formats for the users so that users can check their model's notation more easily. Therefore, the system utilizes the framework and helps users to check their notation usage. The system automatically converts inputted result of requirement analysis to OPM and OPL, and provides natural language expression of their model.

\subsubsection{Automatic monitoring and feedback method of conversation amount}

As mentioned before, this system provides the function to monitor and visualize conversation automatically during workshop because the balanced conversation is one of the most impactful factors for group performance. The overview of the system is shown in Figure 3. To capture conversation, small microphones are introduced in front of the people working in the workshop. Raspberry pi, card-sized computer gathers the microphones' data and send it to data server in real time. The system's user interface also gathers log data of modeling itself which includes actions and the timestamp. By combining these data, the system keep track of who made conversation and what timing and visualize the information.

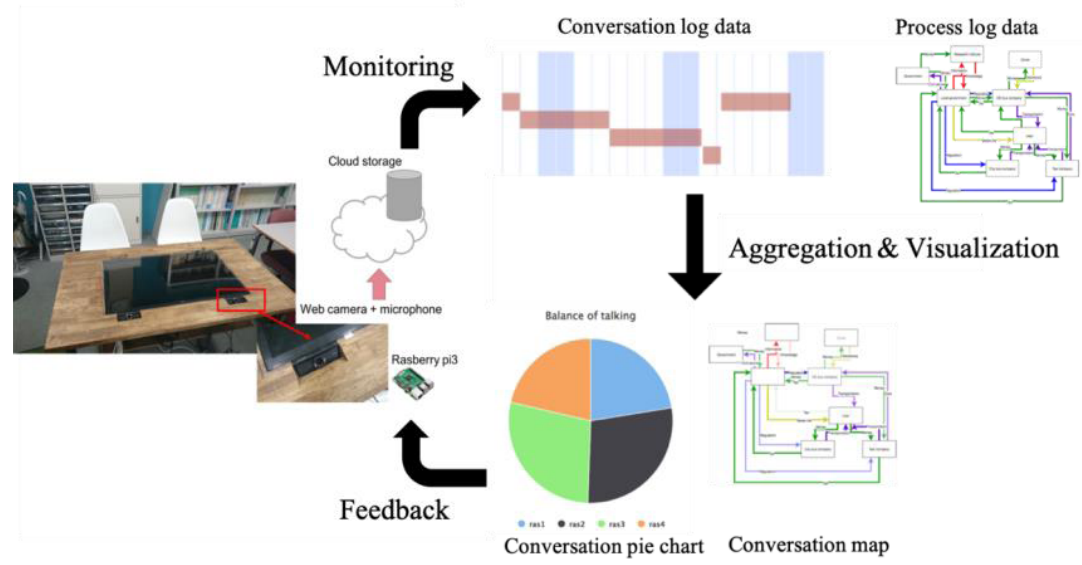

Figure 3. Overview of monitoring and feedback system for balanced conversation. 
The system has 2 ways to visualize the conversation's information, one is just showing percentage of each speaker's conversation, called conversation pie chart, and the other is mapping the information to results of system modeling, we called conversation map. Figure 4 shows an example of the conversation map. The percentage of the member's conversation to each component in the diagram is calculated, and the percentage is mapped on model diagram by color gradation.

\section{Final deliverable: Model diagram}

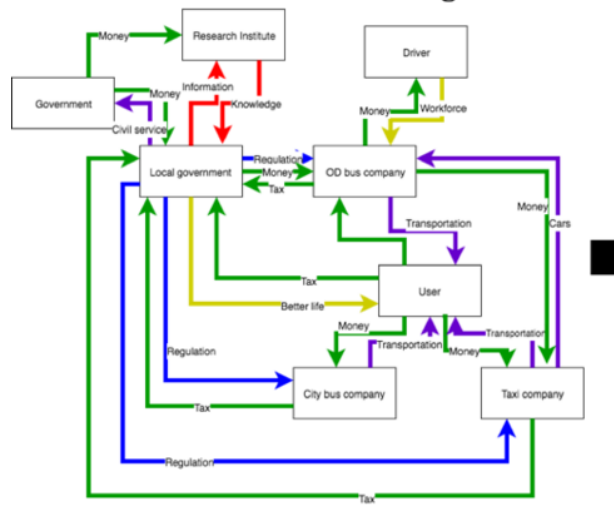

\section{Post analysis: Conversation Mapping}

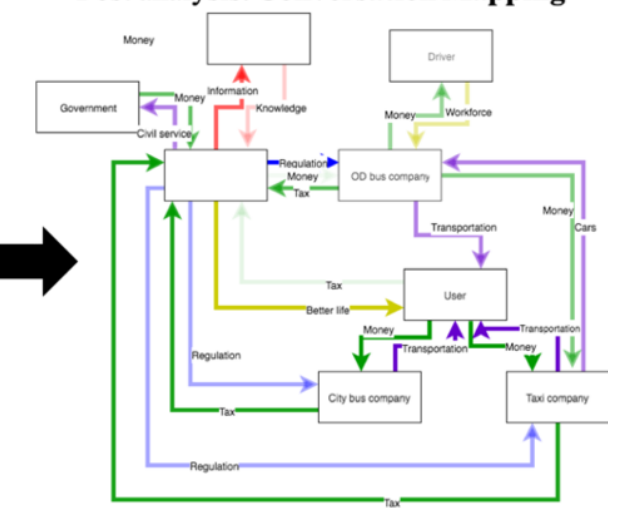

Figure 4. Example of conversation mapping.

This method to visualize conversation assumes that diagram drawing is included as a process in modeling. The calculation of the percentage of contribution is based on 2 kinds of data, one is conversation log and the other is modeling log. Conversation log means log data of conversation in modeling among members and the data can be obtained as audio data. Modeling log is log data of their action in model drawing, for example, adding a text box, adding an arrow, writing text, etc. Modeling log also should include timestamp with those kinds of action log. By connecting these 2 kinds of data, how much conversation is conducted for each action by the members in workshop can be calculated. In more concrete, the percentage for each component in the diagram is calculated by following Equation 1. The equation assumes that all the conversation right before specific action connects to the action and doesn't assume there is any conversation which doesn't connect to the actions. $C_{\text {member }}^{\text {element }}$ is the percentage for each component in the diagram. This element means those components' id, and member is each participant's id. Ti,member is conversation duration automatically detected by the system which is related to element and is spoken by member.

$$
C_{\text {member }}^{\text {element }}=\frac{\sum_{i} T_{i, \text { member }}^{\text {element }}}{\sum_{i} \sum_{\text {member }}\left(T_{i, \text { member }}^{\text {element }}\right)}
$$




\section{Case study}

\subsection{Overview}

The objective of this case is to validate the usefulness of the function to monitor conversation during the collaborative work. A national research institute of ship design and marine transportation is now driving a project in which they are attempting to redesign a large experimental facility for ship design, towing tank and implement it. The project is based on the proposed system design protocol, and as a first step, stakeholder analysis was conducted. The developed system, especially the function of conversation monitoring was applied. In more concrete, it was proceeded with 25 minutes brief guidance of stakeholder analysis, 30 minutes co-working, 10 minutes feedback by the developed system, and 30 minutes co-working. The experimental workshop was conducted by 4 non-experts of system modeling $(\mathrm{P} 1 \sim \mathrm{P} 4)$. It is assumed that all the participants have the same role in the workshop. Therefore, $25 \%$ contribution from each people is the most balanced situation. By observing the workshop before and after the feedback by the developed system, the usefulness of the feedback function is validated.

\subsection{Result}

Figure 5 shows the final deliverable of the workshop. It is originally made in Japanese, and English labels are putted onto the original diagram. 9 stakeholders were enumerated. In the middle of the workshop, the develop system gave them the feedback information including both of conversation pie chart and conversation map.

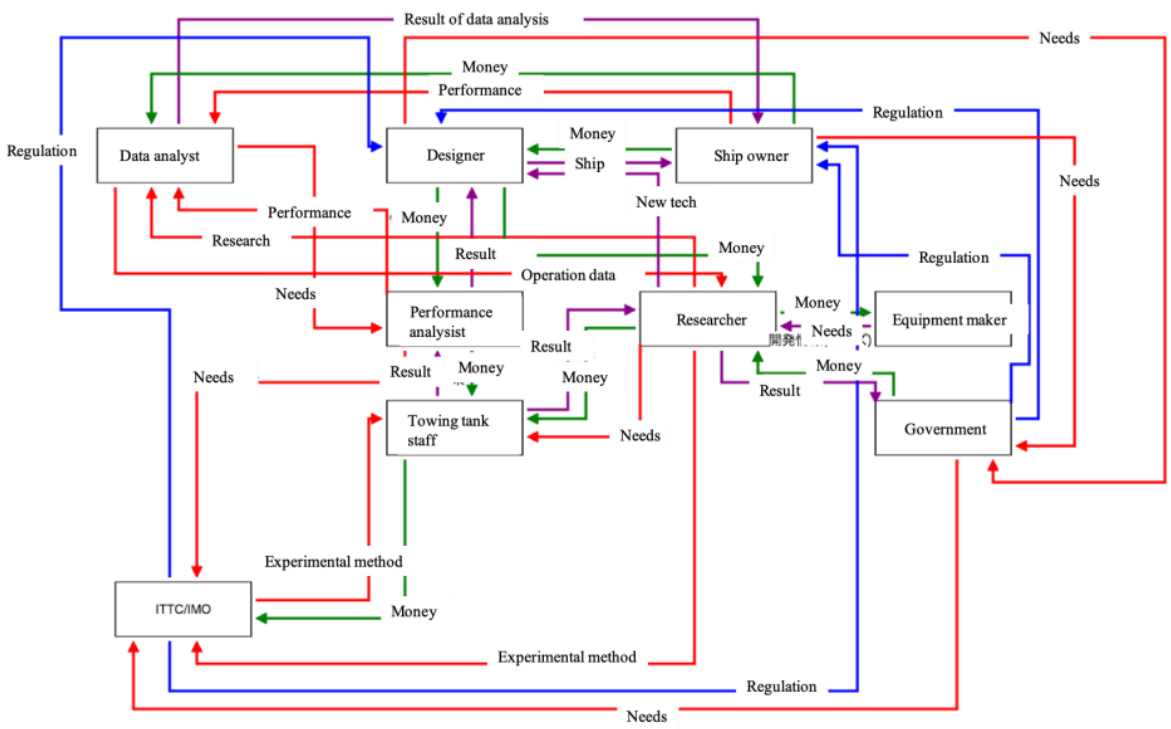

Figure 5. Final outcomes of stakeholder analysis facilitated by the developed system.

Table 1 shows the percentage of amount of conversation by each participant, and how it was affected by the feedback. Before the feedback, participant P1 made more 
than half of total conversation, and leaded the workshop. However, after the feedback, the ratio of $\mathrm{P} 1$ 's conversation was decreased to $44 \%$ of total conversation and the others' conversation was slightly increased. Data quantitatively shows that the balance of distribution of conversation was improved after the system's feedback. And, by analysis of the video data which captures the whole communication during workshop, it is revealed that after the feedback, P1 encouraged the others to share their idea as the workshop and obviously changed his attitude. This encouragement was not observed before the workshop and it qualitatively shows the system's feedback affected his attitude and improved the balance of the conversation. Moreover, some questionnaires were conducted to all the participants to ask the usefulness of the system. They pointed out that the visualization could be a trigger to encourage the other participants who made little conversation to share the idea. And, by the monitoring, they felt they should make a conversation more proactively. These questionnaires' result shows the improvement was caused by the system as well.

Table 1. Changes of the amount of conversation before and after the system's feedback.

\begin{tabular}{lllll}
\hline Participants & Participant1 & Participant2 & Participant3 & Participant4 \\
\hline Before [\%] & 55 & 18 & 17 & 10 \\
\hline After [\%] & 44 & 22 & 20 & 14 \\
\hline
\end{tabular}

Figure 6 shows the result of conversation map in the case. The content is the same as Figure 5, and the depth of color is based on the amount of conversation. The visualization shows not only P1 mainly made conversation but also each participant has different characteristic in the conversation. For example, participant P2 made conversation mainly about policy \& regulation, P4 made it mainly about information exchange among stakeholders. As for participant P3, while the amount of conversation is not so different from the others', the conversation didn't appear on the conversation map. This is because the components he focused during the workshop were removed, and his conversation didn't affect to the final deliverable. Through this observation, it can be said that we can retrieve much more information from the conversation map than simple conversation pie chart.

\section{Conclusion}

The objective of this paper is to develop a collaborative workshop environment for decision-making to improve collaboration in a cross-cultural team. This paper especially approaches 2 kinds of problems, communication among the team members and group performance, and to solve the problems, a concept of instrumented workshop environment was proposed, in which systems approach is utilized to fill the gap of those expertise, and monitoring and automatic visualization system of teamwork was applied to improve the group performance. As a part of demonstration of the proposed environment, an experiment to show the usefulness of the monitoring and visualization system was conducted in the case study. The result of case shows the developed system can trace changes of the conversation amount in the team, and the proposed visualization method, conversation map, clearly projects how much the members made conversation to the workshop's outcome.

Of course, the result comes from just one case and it is needed to conduct a bunch of experiments to make a solid conclusion. However, all the procedures of this 
experiment are facilitated by the information system automatically, and it is repeatable in many times. Future work of this study is to repeat similar experiments, gather enough amount of data, and show the system's usefulness in a statistical mean.
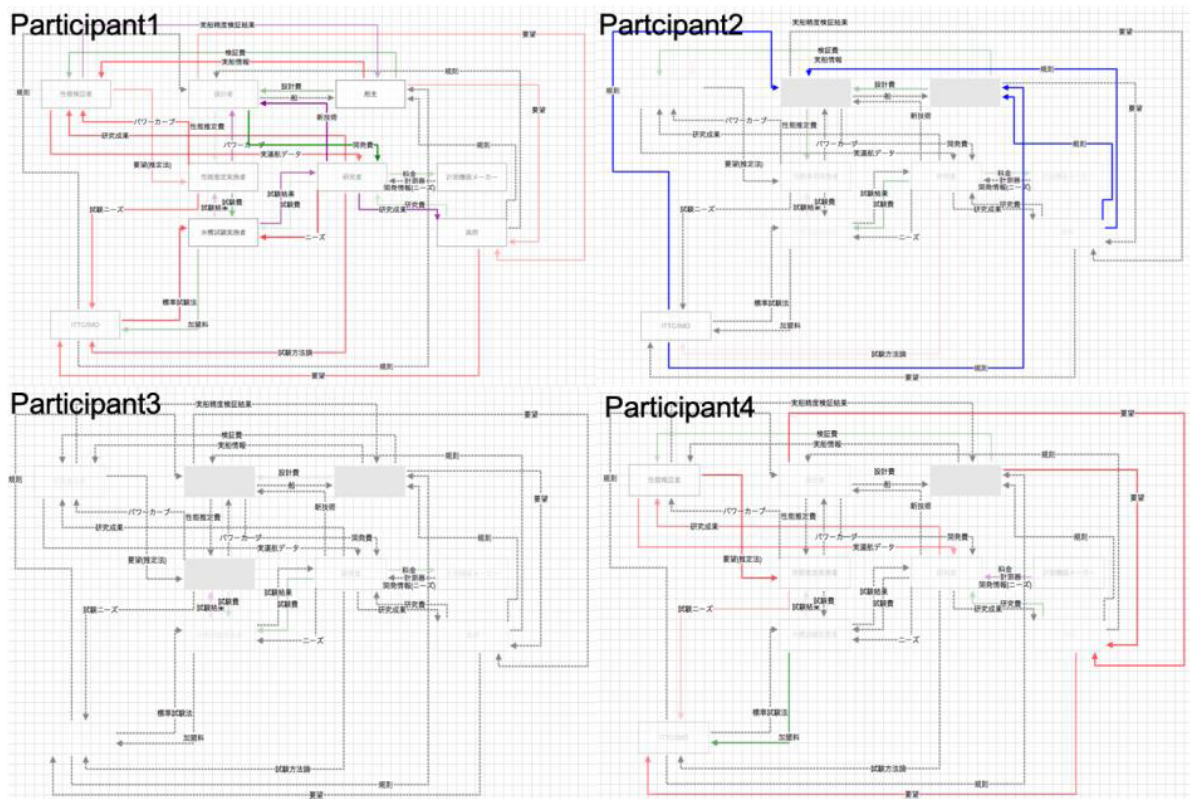

Figure 6. Result of the conversation mapping by the developed system in the case study.

\section{Acknowledgement}

Funding from the Brightline Initiative is gratefully acknowledged.

\section{References}

[1] B.R. Moser and R.T. Wood, Complex Engineering Programs as Sociotechnical Systems. In: J. Stjepandic et al. (eds.) Concurrent Engineering in the 21st Century, Springer Interna., 2015, pp. 51-65.

[2] N.L. Kerr, and R.S. Tindale, Group Performance and Decision Making. Annu. Rev. Psychol., Vol. 55, pp. 623-655, 2004.

[3] D.D. Walden, G.J. Roedler, K. Forsberg, R. D. Hamelin, and T.M. Shortell, Systems Engineering Handbook: A Guide for System Life Cycle Processes and Activities, John Wiley, Hoboken, 2015.

[4] J.E. Gibson, W.T. Scherer, W.F. Gibson and M.C. Smith, How to Do System Analysis: Primer and Casebook, Wiley, Hoboken, 2017

[5] A.W. Woolley, C.F. Chabris, A. Pentland, N. Hashmi and T.W. Malone, Evidence for a Collective Intelligence Factor in the Performance of Human Groups. Science, Vol. 330, 6004, pp. 686-688, 2010.

[6] I.L Janis, Groupthink: Psychological Studies of Policy Decisions and Fiascoes, Houghton Mifflin, Boston, 1982.

[7] C.R. Leana, A Partial Test of Janis' Groupthink Model: Effects of Group Cohesiveness and Leader Behavior on Defective Decision Making. Journal of Management, Vol. 11, No. 1, pp. 5-18, 1985.

[8] B.G. Cameron, E.F. Crawley, G. Loureiro, and E.S. Rebentish, Value Flow Mapping: Using Networks to Inform Stakeholder Analysis, Acta Astronautica, Vol. 62, pp. 324-333, 2008.

[9] G. A. Miller, WordNet: A Lexical Database for English, Communications of the ACM, Vol. 38, No. 11, pp. 39-41, 1995.

[10] D. Dori, Model-Based Systems Engineering with OPM and SysML, Springer, New York, 2016. 